\title{
Applying Topsis in Agent-based Dynamic Supply Chain Partner Selection
}

\author{
Cao Mukun \\ School of Management \\ Xiamen University \\ Fujian, Chain
}

\author{
ZhaoYang \\ School of Management \\ Xiamen University \\ Fujian, Chain
}

\begin{abstract}
Partner selection is an important step of the supply chain companies. This issue belongs to the multi-attribute Decision-making Problem. This work is based on the framework of MAS-based dynamic supply chain. The basic idea of Agent is to make the software to simulate organizational behavior and relationships,so using agent to select the partner is a trend. It applies TOPSIS method in the partner selection. The feature of this study is how to deal with the interval attribute in partner selection. Finally, this work uses an examples to illustrate how Enterprise Agent to select thepartners that the attributes are intervalin the partner selection.
\end{abstract}

Keywords-Dynamic supply chain,Partner selection, TOPSIS (Technique for order preference by similarity to ideal solution), MAS(Multi-Agents)

\section{INTRODUCTION}

To survive in the competitive, cooperative, dynamic market environment, enterprise implementing the dynamic supply chain management is imperative. Dynamic supply chain consists of suppliers, manufacturers and distributors who cooperate to achieve a specific purpose. After their aims achieve, they can decide to end their relationship and then wait for the next cooperation with other companies to make up a new supply Chain[1]. These enterprises are called as partners in the business process. Each partner in their respective superiority areas (such as design, manufacturing, distribution, etc.) contributes their core competencies for the union. They combine together to achieve mutually complementary advantages and risks and benefits sharing. Dynamic supply chain requires that each business entities as well as supply chain have a rapid response to changing market conditions, and the ability of a good partnership can improve the efficiency and competitiveness of the entire supply chain. To achieve the win-win situation, it is important to establish an objective, scientific and comprehensive mechanism for the efficient partner selection. Therefore, the problem of the dynamic supply chain partner selection has practical significance.

Thesupply chain partner selectionhas some characteristics of high integration of information, wide distribution, fast changeand heterogeneous. It isnecessary to search out many partners to meet the requirements of enterprisesand evaluate each candidate.According to the characteristics of their own production, select the appropriate partner[2]. With the increasing competitions within and outside the supply chain, the quality and efficiency of supplier selectionhas a critical influence on performanceof the supply chain. The basic idea of Agent is to make the software to simulate organizationalbehavior and relationships[3].Its status can be seen as the belief, capability, select, commitment and other components made of mind. They can be perceptive and cooperative each other, which can collaborate to solve the task.

Agent has the feature of autonomy, sociality and learning. Therefore, using Agent in the partner selection process, we can improve the accuracy, objectivity and efficiency of partner selection.In a MAS-baseddynamic supply chain system, each Agent represents different organization. Due to certain interests, they cooperate with each other to finish processing, assembling and distribution.

The risk of dynamic supply chain partner selection is higher than it of stable supply chain, so the choice of partners is particularly important. Many scholars discussed this issue in the previous literatures, and raise some methods to decide how to select the partners: data envelopment analysismethod (DEA)[4], principal component analysismethod (PCA)[5], fuzzy evaluation method[6,7], analytic hierarchy process (AHP)[8], rough set evaluationmethod[9], genetic algorithm[10], method based on indicators of balance and weight interval[11], gray model method[12], fuzzy programming method[13] and so on, but these methods can not solve these supplier selection problems that the attributes are interval.This literature proposes to apply TOPSIS method inselecting partners of MAS-based dynamic supply chain. This method can handle the intervalattributes, using an integrated decision of qualitative and quantitative methods.

II. THE FRAMEWORK OF MAS-BASEDDYNAMIC SUPPLY CHAIN

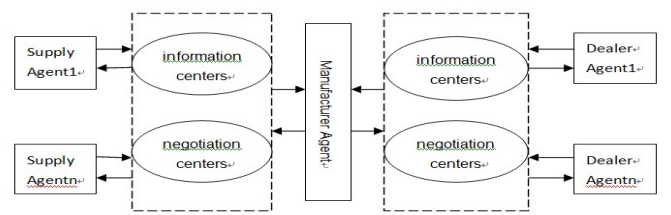

Figure 1. the framework of MAS - based dynamic supply chain

Manufacturer agent is the core of enterprise supply chain. Its main role is products management, conducting business, integrating business functions and information model in the base of enterprise resource planning system. When they do not have enough partners, manufacturers Agent selects appropriate partner in the information center, consults with each other in the negotiation center, and achieves a long-term strategic cooperation after a period of evaluation. 
The main function of suppliers Agent and dealer Agent is automatically negotiating with the manufacturer Agent online to achieve the purpose of strategic cooperation. In the stage of strategic cooperation, the suppliers Agent and dealer Agent provide their management information to the manufacturer as much as possible, in order to optimize supply chain and improve supply chain efficiency.

Negotiation center provides consultation services for the supply chain management, such as building the appropriate standards of communication and consultation channels between manufacturers and suppliers. Communicating among members of the Agents, it is necessary to use some standard knowledge, data, and information communication mode. Therefore, the negotiation center sets up a rule server to establish normative knowledge representation for each field, and to provide the standards of the language communications and relevant knowledge such as definition, attributes, relationships, constraints and other aspects.

Information Center is responsible for receiving, registering and recording the service information in the database, and other Agents will query the information and service center to determine which Agent can provide what kind of service and extract the location of these Agents and other related information[16]. As the dynamics and complexity of the supply chain, the manufacturers need to check the enterprises which can provide raw materials, outsourcing services, transportation, distribution and retail. Therefore, the information center plays an important role to provide search services.

\section{The BASIC PRINCIPLES AND Methods Of Topsis}

\subsection{THE PRINCIPLE OF TOPSIS}

TOPSIS method is based on the concept of the shortest distance between the selected program and the positive point. Because assuming each attribute monotonically increasing or decreasing, it can be transformed into Euclidean distance to be calculated and measured, and obtained the best program by the comprehensive comparison. The so-called positive points is to imagine the best solution, its target value of each candidate plan achieves the best value; and the negative point is the solution envisaged the worst, its target value of each candidate plan achieves the worst value. The basic idea of TOPSIS method is that simultaneously consider the distances from each program point to the positive point and negative point respectively. The appropriate point should be near as much as possible from the positive solution, and be far away from the negative point as much as possible. If one of the candidate plan which is the closest to the positive point but the farthest away from the negative point, this solution is the best program of concentrated solution, which is the final solution.

\subsection{ANALYSIS AND SELECTION PROCEDURES}

With the social and economic development, the complexity and uncertainty of questions and the ambiguity of the human mind is constantly enhanced. The attribute values have three forms in Multi-attribute decisions: a real number, interval number and language. This paper is based on the interval attribute, because choosing the partners in the reality, many attribute values are not uncertain but a range.
We can use the interval numbers and other information to rank the candidates, and further help to make the appropriate decisions. Specific steps are as follows:

Step 1 For the multi-attribute decision making problems, suppose the attribute weights are completely determined, $\tilde{A}=\left(\tilde{a}_{i j}\right)_{n \times m}$ express as a decision matrix, $R=\left(r_{i j}\right)_{n \times m}$ express

as a normalized matrix.

Step 2 Use the attribute weights $w$ and normalized matrix $\tilde{R}$,construct the weighted normalized decision matrix $\tilde{Y}=\left(\tilde{y}_{i j}\right)_{n \times m}$

Step 3 Use (4.16) and (4.20) to determine the positive points $\tilde{y}^{+}$and negative

$$
\begin{aligned}
& \text { points } \tilde{y} \text {. } \\
& \tilde{y}_{j}^{+}=\left[\begin{array}{ll}
\tilde{y}^{+} & \tilde{+}^{+} \\
y & , y
\end{array}\right]=\left[\max _{i}\left(y_{i j}^{L}\right), \max _{i}\left(y_{i j}^{U}\right)\right](j=1,2, \ldots, \quad m) \\
& \tilde{y}_{j}^{-}=\left[\begin{array}{ll}
\tilde{y}^{-L} & \tilde{y}^{-U} \\
& y
\end{array}\right]=\left[\min _{i}\left(y_{i j}^{L}\right), \min _{i}\left(y_{i j}^{U}\right)\right]\left(\begin{array}{ll}
j=1,2, \ldots, & m
\end{array}\right)
\end{aligned}
$$

Step 4 Calculate the distances from each program point to the positive point and negative point respectively.

$$
\begin{aligned}
& D_{i}^{+}=\sum_{j=1}^{m}\left\|\tilde{y}_{i j}-\tilde{y}_{j}^{+}\right\|=\sum_{j=1}^{m}\left[\left|y_{i j}^{L}-y_{j}^{+L}\right|+\left|y_{i j}^{U}-y_{i j}^{+U}\right|\right] \\
& (i=1,2, \ldots, n) \\
& D_{i}^{-}=\sum_{j=1}^{m}\left\|\tilde{y}_{i j}-\tilde{y}_{j}^{-}\right\|=\sum_{j=1}^{m}\left[\left|y_{i j}^{L}-y_{j}^{-L}\right|+\left|y_{i j}^{U}-y_{j}^{-U}\right|\right] \\
& (i=1,2, \ldots, n)
\end{aligned}
$$

Step 5 Calculate ${ }^{c_{i}}$ of each program to the ideal point.

$$
c_{i}=\frac{D_{i}^{+}}{D_{i}^{+}+D_{i}^{-}}(i=1,2, \ldots, n)
$$

Step 6 Rank the value of $c_{i}$, the value is larger, the program is better.

\section{ApPly TOPSIS Multi-AtTRIBUte Decisions} Method In DyNAMIC SUPPly CHAin PARTNER SELECTION

Dynamic supply chain partner selection is always in the form of one to many. In one to many agent negotiations, one side has the right to choose the partner from many candidates. Before the negotiation, the manufacture agent broadcasts the necessary attributes to the candidate agents, and determines each attribute weight by itself. The candidate agents do not know the attribute weight. The candidate agents notify the manufacture agent the range of every attribute. The range is from the acceptable minimum to maximum. Accordingly, the attribute of each candidate agent forms an interval. There is no communication among the candidate agents. Then apply the TOPSIS method in each candidate agents, and rank them to find a more satisfied partner.

The following example occurs between five raw material suppliers and a manufacturer. The manufacturer requires that 
the raw material should arrive once and have no difference in quality, so it selects just one raw material supplier as negotiating partner.

Manufacturer Agent requires the five material providers Agent to publish the scope of their time, cost, defect rate and credit evaluation, only to manufacturer. The attributes of time, cost and defect rate are the cost-based, its value should be as small as possible; the credit evaluation attribute is benefit-based, its value should be as large as possible. The credit evaluation is based on the score of other partners evaluating the supplier in previous cooperation. The score includes quality reputation, financial reputation and social reputation[17], whose value is from 0 to 10 (the evaluationscore table shown in Tab.1). During this period, only the manufacturer agent knows the information of the five material supply Agents, but each of the five don't know other information and form an alliance.

When the raw material supply Agents report the relevant information to the manufacture Agent, which includes: U1cost (ten thousand); U2-time (days); U3-defect rate (\%); U4credit evaluation (Value range from 0 to 10), the manufacturer Agent will aggregate information into a decision matrix

\section{$\tilde{A}$, as shown in Tab.2:}

Step 1 The attributes designed in the negotiations are fully ascertainable (real numbers). Agi is short for Agenti, whose attributes value are measured by $\tilde{a}_{i j}=\left[a_{i j}^{L}, a_{i j}^{U}\right]$, which constitute the

decision matrix $\tilde{A}=\left(\tilde{a}_{i j}\right)_{n \times m}$. The most common attributes are benefit type and cost type.

$I_{1}$ represents benefit type and $I_{2}$ represents cost type. In order to eliminate the influence of different physical dimensions in the decision-making, the following formula can transform the decision

$$
\begin{gathered}
\text { matrix } \tilde{A} \text { into the normalized decision } \\
\operatorname{matrix} \tilde{R}=\left(\tilde{r}_{i j}\right)_{n \times m}, \tilde{r}_{i j}=\left[r_{i j}{ }^{L}, r_{i j}{ }^{U}\right]
\end{gathered}
$$

Benefit-based attribute:

$$
\left\{\begin{array}{l}
r_{i j}^{L}=\frac{a_{i j}^{L}}{\sqrt{\sum_{i=1}^{n}\left(a_{i j}^{U}\right)^{2}}} \quad i=1,2, \ldots, \quad n, j \in I_{1} \\
r_{i j}^{U}=\frac{a_{i j}^{U}}{\sqrt{\sum_{i=1}^{n}\left(a_{i j}^{L}\right)^{2}}}
\end{array}\right.
$$

Cost-based attribute:

$$
\left\{\begin{aligned}
r_{i j}{ }^{L} & =\frac{\frac{1}{a_{i j}^{U}}}{\sqrt{\sum_{i=1}^{n}\left(\frac{1}{a_{i j}^{L}}\right)^{2}}} i=1,2, \ldots, n, j \in I_{2} \\
r_{i j}^{U} & =\frac{\frac{1}{a_{i j}^{L}}}{\sqrt{\sum_{i=1}^{n}\left(\frac{1}{a_{i j}^{U}}\right)^{2}}}
\end{aligned}\right.
$$

Substituting the corresponding data to calculate the normalized decision matrix $\tilde{R}$ (to retain two significant figures), as shown in Tab.3:

Step 2 The manufacturer agent sets each attribute weight vector $w=(0.27,0.23,0.26,0.24)$,

calculates the weighted normalized decision matrix

$\tilde{Y}$, as shown in Tab.4:

Step 3 Use the formula (1) and (2) to determine the positive point $\tilde{y}^{+}$and negative

$$
\begin{aligned}
& \tilde{\sim}^{-} \\
& \text {point } \tilde{y}^{+}: \\
& \tilde{y}^{-}=([0.12,0.15],[0.08,0.19],[0.12,0.18],[0.11,0.13]) \\
& \tilde{y}^{-}=([0.10,0.12],[0.06,0.10],[0.07,0.10],[0.08,0.10])
\end{aligned}
$$

Step 4 According to the formula (3) and (4), calculate the distances from each program point to the positive point and negative point respectively:

$$
\begin{aligned}
& D_{1}^{+}=0.18 ; D_{2}^{+}=0.10 ; D_{3}^{+}=0.21 ; D_{4}^{+}=0.19 ; D_{5}^{+}=0.18 \\
& D_{1}^{-}=0.18 ; D_{2}^{-}=0.19 ; D_{3}^{-}=0.14 ; D_{4}^{-}=0.18 ; D_{5}^{-}=0.19
\end{aligned}
$$

Step 5 According to the formula (5), calculate $c_{i}$ of each program to the ideal point:

$$
c_{1}=\frac{D_{1}^{+}}{D_{1}^{+}+D_{1}^{-}}=\frac{0.18}{0.18+0.18}=0.50, c_{2}=0.34, c_{3}=0.60, c_{4}=0.51, c_{5}=0.49
$$

Step 6 Rank the value of $c_{i}$, draw a conclusion: $c_{3} \succ c_{4} \succ c_{1} \succ c_{5} \succ c_{2}$.

After calculation, the manufacturer makes a preliminary decision to select the raw material provider. The manufacture Agent can choose to negotiation with Ag3 . If successful, reach a cooperation agreement; if not successful, recalculate the above steps excluding Agent3, until the manufacture agent negotiates with a raw material supplier successfully.

\section{SUMMARIES}

Dynamic supply chain partner selection has always been a hot topic in academia. In practice, because of the complexity and uncertainty of questions and the ambiguity of the human mind, we always meet the interval attributes. In this example, each agent has meritsanddemerits, so we cannot decide which agent is theappropriate one. This work applied TOPSIS method in dynamic supply chain partner selection instead of selectingsubjectively, and deals with the interval attributes. In future, you can consider how to apply TOPSIS in the case that the interval value is continuous and discontinuous co-exist.acknowledgement 
This work is supported by grant 2009J05162 of the China Fujian Province Natural Science Foundation, and by grant 70902042 of the National Natural Science Foundation of China (NSFC).

\section{REFERENCES}

[1] SU Shi-bin; YANG Mao-sheng. Select Cooperative Partners for Dynamic Chain Based on Extension AHP.Logistics Technology[J]. 2005(11),PP:55 57.

[2] MA Shi-hua; LIN Yong; CHEN Zhi-xiang. Supply Chain Management[M]. Beijing: China Machine Press,2000.

[3] WU Bei-ni; ZHOU Liang; Ling Xing-hong. Agent-based Supply Chain Partnership Estimate System. Application Research of Computers[J].2003(3),PP:38 39.

[4] SUNJing-chun; Wang Kan-liang; Su Qin.Measuring Decision Making For Vendor Combination with DEA.High Technology Letters[J].2000(11),PP:53 56.

[5] PetroniA;BragliaM.Vendorselection using principal component analysis.Supply Chain Management[J].2000(36),PP:63 69.

[6] Zhang Bing-xuan;Li Long-zhu;Du Zhong-cheng.The Evaluation System and Fuzzy Evaluation Method for Dynamic Supply Chain.Journal of Tianjin Normal University[J].2001(3).PP:19 23.

[7] Wang Fang;Wu Qi-zong. Fuzzy Multiple Attribute Decision Making under Incomplete Attribute Weights Based on Cooperation Partner of Supply Chain Selection.Mathematics in Practice and Theory[J].2008(24),PP:18 24.

[8] Tam,Maggie C.Y;Tummala,V.M.Rao.An application of the AHP in vendor selection of a telecommunications system. International Journal of Manage Science [J].2001(29),PP:171 182.
[9] Cao Xiu-ying; Liang Jing-guo.An Application of the Rough Set Evaluation Method in Supply Chain Partner Selection.Science of Science and Management of Science \& Technology[J]. 2002(8),PP:113 115.

[10] Ma Zu-jun.Partner Selection of Supply Chain Alliance Based on Genetic Algorithms. Systems Engineering-Theory \& Practice [J]. 2003(9),PP:81 84.

[11] Gao Yang; Li Yi-hua.A Method for Partner Selection in Supply Chain Based on Index Equilibrium and Power Interval Numbers.Operations Research and Management Science [J]. 2004(4),PP:155 159.

[12] Liu Hai-jin, Zhang Ying-min. Application of Grey Correlation Model in the Selection of the Partners of the Supply Chain. Logistics Science and Technology[J].2006(136),PP:54 56.

[13] ManojKumar; PremVrat; Ravi Shankar.A Fuzzy Programming Approach for Vendor Selection Problem in a Supply Chain.International Journal of Production Economics [J]. 2006(101),PP: 273- 285.

[14] MA Shi-hua. Solution for Supporting Operations Management of Supply Chain.Computer Integrated Manufacturing Systems[J]. 2002(8),PP:630 634.

[15] LIU Guang-zhong, SHI Xiao-long.Agent Management Model and Architecture.Computer Engineering.2002(2),PP:91 92.

[16] CHEN Jian, FENG Wei-dong.Construction and Management of Virtual Enterprise. Beijing: Tsinghua University Press, 2000.

[17] Yang Jie; Liu Yin;Sun Li-peng.Web-based Supply Chain Partnership Selection for Agile Manufacturing.Journal of Wuhan University of Technology.2004(3),PP:424 426.

TABLE I. EVALUATION-SCORE TABLE

\begin{tabular}{|c|c|c|c|c|c|}
\hline evaluation & worst & worse & normal & better & best \\
\hline score & $0 \sim 2$ & $3 \sim 4$ & $5 \sim 6$ & $7 \sim 8$ & $9 \sim 10$ \\
\hline
\end{tabular}

TABLE II. DECISION MATRIX

\begin{tabular}{|c|c|c|c|c|}
\hline$\tilde{A}$ & Cost-U1 & Time-U2 & defect rate-U3 & $\begin{array}{c}\text { credit } \\
\text { evaluation-U4 }\end{array}$ \\
\hline Agent1 & {$[40,45]$} & {$[10,12]$} & {$[1.5,1.9]$} & {$[8.0,9.0]$} \\
\hline Agent2 & {$[35,40]$} & {$[5.0,8.0]$} & {$[2.7,3.1]$} & {$[9.0,9.5]$} \\
\hline Agent3 & {$[42,45]$} & {$[7.0,9.0]$} & {$[1.8,2.0]$} & {$[7.0,8.0]$} \\
\hline Agent4 & {$[35,38]$} & {$[7.0,10]$} & {$[2.5,2.8]$} & {$[9.0,10]$} \\
\hline Agent5 & {$[40,45]$} & {$[6.0,9.0]$} & {$[2.0,2.5]$} & {$[8.0,9.0]$} \\
\hline
\end{tabular}

TABLE III. NORMALIZED DECISION MATRIX

\begin{tabular}{|c|c|c|c|c|}
\hline$\tilde{R}$ & \multirow{2}{*}{ U1 } & U2 & U3 & U4 \\
\hline $\mathrm{Ag} 1$ & {$[0.37,0.48]$} & {$[0.24,0.42]$} & {$[0.46,0.70]$} & {$[0.39,0.49]$} \\
\hline $\mathrm{Ag} 2$ & {$[0.42,0.50]$} & {$[0.36,0.84]$} & {$[0.28,0.39]$} & {$[0.44,0.52]$} \\
\hline $\mathrm{Ag} 3$ & {$[0.37,0.46]$} & {$[0.32,0.60]$} & {$[0.44,0.58]$} & {$[0.34 .0 .43]$} \\
\hline $\mathrm{Ag} 4$ & {$[0.44,0.56]$} & {$[0.29,0.60]$} & {$[0.31,0.42]$} & {$[0.44,0.54]$} \\
\hline $\mathrm{Ag} 4$ & {$[0.37,0.48]$} & {$[0.32,0.70]$} & {$[0.35,0.52]$} & {$[0.39,0.49]$} \\
\hline
\end{tabular}

TABLE IV. THE WEIGHTED NORMALIZED DECISION MATRIX

\begin{tabular}{|c|c|c|c|c|}
\hline$\tilde{Y}$ & U1 & U2 & U3 & U4 \\
\hline $\mathrm{Ag} 1$ & {$[0.10,0.13]$} & {$[0.06,0.10]$} & {$[0.12,0.18]$} & {$[0.09,0.12]$} \\
\hline $\mathrm{Ag} 2$ & {$[0.11,0.14]$} & {$[0.08,0.19]$} & {$[0.07,0.10]$} & {$[0.11,0.12]$} \\
\hline $\mathrm{Ag} 3$ & {$[0.10,0.12]$} & {$[0.07,014]$} & {$[0.11,0.15]$} & {$[0.08,0.10]$} \\
\hline $\mathrm{Ag} 4$ & {$[0.12,0.15]$} & {$[0.07,0.14]$} & {$[0.08,0.11]$} & {$[0.11,0.13]$} \\
\hline $\mathrm{Ag} 4$ & {$[0.10,0.13]$} & {$[0.07,0.16]$} & {$[0.09,0.14]$} & {$[0.09,0.12]$} \\
\hline
\end{tabular}

